\title{
Arcadia5. Arquitectura y hábitat. Soberanía de las
}

\section{PERSONAS}

\section{Arcadia5. Architecture and Hábitat. Popular Sovereignty}

\author{
Alex Mitxelena Etxeberria ${ }^{1}$ et al. ${ }^{2}$ \\ Fecha de recepción: 13-06-2019 - Fecha de aceptación: 10-09-2019 \\ Hábitat y Sociedad (ISSN 2173-125X), n. ${ }^{\circ}$ 12, noviembre de 2019, pp. 135-142. \\ http://dx.doi.org/10.12795/HabitatySociedad.2019.i12.09
}

\begin{abstract}
Arcadia5 was held on October 18 and 19, 2018 in the E.T.S. of Architecture of the UPV / EHU in San Sebastián. In this edition of the congress the objective was to continue contributing to an important corpus of knowledge that should be part of the architecture schools, forming new architects capable of looking at the world, from the local and the small, but without forgetting the global and how we are interconnected with others; focusing on people and their needs, and also without neglecting the environment. This time the Arcadia Congress on Architecture and Development Cooperation put the focus on the people who inhabit the planet in order to achieve an architecture for people, that is, that responds to the needs, experiences and dreams of those who live in the villages, the houses, the towns and the cities, with their singularities, characteristics and cultural and identity differences.
\end{abstract}

Key words

Architecture; Cooperation; Development; People; Sovereignty

\section{Resumen}

Arcadia5 se celebró los días 18 y 19 de octubre de 2018 en la E.T.S. de Arquitectura de la UPV/EHU en San Sebastián. En esta edición del congreso el objetivo fue seguir contribuyendo a un importante corpus de conocimiento que debe de ser parte de las escuelas de arquitectura, formando nuevxs arquitectxs capaces de mirar al mundo, desde lo local y lo pequeño, pero sin olvidarse de lo global y de cómo estamos interconectadxs unxs con otrxs; centrándose en las personas y sus necesidades, y además sin descuidar el medio ambiente. Esta vez el Congreso Arcadia sobre Arquitectura y Cooperación al Desarrollo puso el foco en las personas que habitan el planeta con el fin de conseguir una arquitectura para las personas, es decir, que responda a las necesidades, vivencias y sueños de quienes habitan los pueblos, las casas, las aldeas y las ciudades, con sus singularidades, características y diferencias culturales e identitarias.

Palabras clave

Arquitectura; Cooperación; Desarrollo; Personas; Soberanía

\footnotetext{
1 Dr. Arquitecto, profesor del Área de Proyectos Arquitectónicos. Departamento de Arquitectura de la UPV/EHU, Donostia, España. E-mail: alex.mitxelena@ehu.eus

2 Izaskun Aseguinolaza Braga (Dra. Arquitecta, profesora del Área de Urbanística y Ordenación del Territorio. Departamento de Arquitectura de la UPV/EHU. ETS Arquitectura, Donostia, España. E-mail: izaskun.aseguinolaza@ehu.eus); Madalen González Bereziartua (Dra. Arquitecta, profesora del Área de Urbanística y Ordenación del Territorio. Departamento de Arquitectura de la UPV/ EHU, ETS Arquitectura, Donostia, España. E-amil: madalen.gbereziartua@ehu.eus); Ramón Barrena Etxebarria (Arquitecto, profesor del Área de Proyectos Arquitectónicos. Departamento de Arquitectura de la UPV/EHU, Donostia, España. E-mail: ramon.barrena@ehu.eus); Koldo Tellería Andueza (Arquitecto, profesor del Área de Urbanística y Ordenación del Territorio. Departamento de Arquitectura de la UPV/EHU. ETS Arquitectura, Donostia, España. E-mail: koldo.telleria@ehu.eus); Enkarni Gómez Genua (Dra. Ingeniera, profesora del Departamento de Física Aplicada I de la UPV/EHU. ETS Arquitectura, Donostia, España. E-mail: enkarni.gomez@ehu.eus).
} 
El Congreso Arcadia sobre Arquitectura y Cooperación al Desarrollo se celebra bianualmente en diferentes escuelas de arquitectura de España. En su quinta edición, Arcadia5 se celebró los días 18 y 19 de octubre de 2018 en la E.T.S. de Arquitectura de la UPV/EHU en San Sebastián, ${ }^{3}$ donde anteriormente se habían desarrollado diferentes proyectos relacionados con la temática. En particular, el PIE (Proyecto de Investigación Educativa) titulado "La Cooperación al Desarrollo como marco para la adquisición de competencias transversales en el Grado en Fundamentos de Arquitectura" (Gómez, Mitxelena y Aseguinolaza, 2017) se realizó durante los cursos 2014-16 reuniendo un grupo de profesorxs ${ }^{4}$ convencidxs de que "la cooperación al desarrollo es parte esencial del compromiso social de la Universidad y como tal no puede entenderse como una actividad extraacadémica ni restringida a compromisos individuales, sino integrada en el conjunto de actividades que le son propias", tal y como se recoge en el artículo 12 del Código de Conducta de las Universidades en materia de Cooperación al Desarrollo (CEURI, 2006).

Como decimos, este hito consolida en nuestra Escuela una línea de trabajo llevada a cabo en torno a la cooperación al desarrollo como son el programa de la Universidad del País Vasco denominado Gaztenpatia y el anterior Proyecto maCOOa (Pennese y Mitxelena, 2015). Es en este contexto en el que la Escuela de Arquitectura de la UPV/EHU recibe el testigo en A Coruña para organizar la siguiente edición del encuentro Arcadia: Arcadia5.

El comité organizador de la E.T.S. de Arquitectura de la UPV/EHU de Arcadia5 continúa el trabajo de las ediciones anteriores ${ }^{5}$ y en la línea ya establecida en la Escuela del País Vasco. Esta vez se ha puesto el foco en las personas que habitamos este planeta, en una arquitectura que actúa como eje vertebrador de las relaciones entre las personas, que responde a las necesidades, vivencias y sueños de quienes habitan los pueblos, las casas, las aldeas y las ciudades, con sus singularidades, características y diferencias culturales e identitarias.

El lugar que habitamos condiciona nuestra forma de vida. Su presencia es inevitable y constante, sin embargo, muchas veces no somos conscientes de en qué medida es un aliado o una barrera. Por ello, el eje temático de este Congreso Arcadia5 se centró en los espacios o hábitats que favorecen la soberanía de las personas.

Se trata de promover una arquitectura que requiere de un proceso

3 Para profundizar sobre el origen del Congreso: cfr. De Manuel Jerez, Esteban y Martín Hernández, Manuel J. (2010). Jornadas de Arquitectura y Cooperación al Desarrollo. Hábitat y Sociedad, 1, 175-179. 4 NOTA A LX LECTORX: Queremos aclarar que en el uso de un lenguaje inclusivo lxs autorxs hemos elegido emplear el carácter "x". Desde nuestro punto de vista, este carácter tiene la función de incluir a todxs lxs individuxs posibles, en su diversidad de géneros y sexos ya que, por un lado, es el carácter utilizado como variable matemática de una ecuación que cada unx debe resolver y cuyo resultado siempre es diferente y es, además, el signo de la multiplicación.

5 En la web de Arcadia 5, http://www. arcadia5.eus/, hemos recogido la documentación que se puede encontrar en la red sobre las anteriores ediciones. integrador, que permita el trabajo conjunto de profesionales, diseñadorxs, usuarixs y ciudadanía y que enriquezca el resultado por su complejidad y diversidad. Este tipo de trabajo participativo y en grupo favorecerá, a su vez, la soberanía de todxs lxs participantes e implicadxs (Figura 1).

Para abordar estos temas, se organizaron cuatro módulos, cada uno referido a un enfoque que a continuación se resume.

En el módulo 1, se propuso una reflexión en torno al medio urbano, que en muchas ocasiones no responde a los usos que resultan necesarios para atender a la vida cotidiana de las personas. Gran parte del espacio urbano de las ciudades está en manos del coche; depende de fuerzas económicas cuyos intereses son, muchas veces, ajenos a la ciudadanía; adolece de una respuesta al entorno natural; y ha sido diseñado para un individuo genérico que no representa la diversidad social existente. Por ello, la ciudad tiene, actualmente, un reto fundamental: recuperar el espacio urbano unido al entorno natural para la ciudadanía diversa y compleja. 


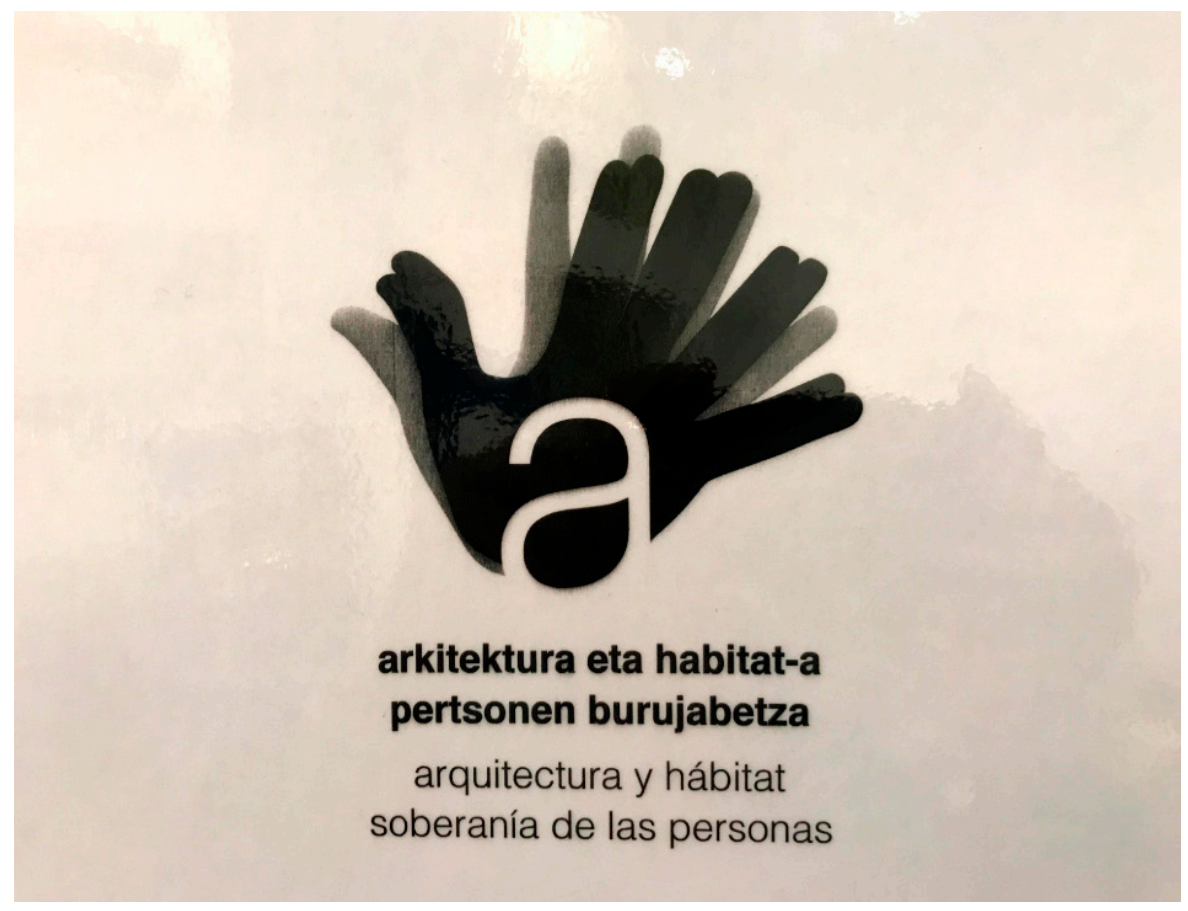

En el módulo 2, la discusión giró en torno al medio humano, cuyo reto perseguía afrontar las consecuencias de una globalización que conduce a la pérdida de la identidad cultural de cada territorio y en el que se propuso dirigir las contribuciones hacia la recuperación y fortalecimiento de estas identidades.

En el módulo 3, ligado a la escala de las edificaciones y la construcción, se propuso reflexionar sobre la arquitectura y cómo esta puede facilitar la relación entre las personas y la relación de estas con las construcciones, el entorno y el contexto urbano y natural. Cuando se incorporan las necesidades humanas, su identidad individual y colectiva, decimos que la arquitectura "pone a las personas en el centro". En esta escala se necesitan propuestas que respondan a las necesidades de sus habitantes, reconociendo su diversidad y complejidad, sabiendo que estas cambian a lo largo de la vida. En este módulo, se quiso poner en valor la arquitectura pensada para las personas; espacios que ponen en el centro el cuidado mutuo, el respeto y la convivencia; la arquitectura en mayúsculas, aquella que celebra la vida de quienes la utilizan y la habitan.

Finalmente, en el módulo 4 se planteó el contexto de la educación para el desarrollo. Vivimos en un contexto social en el que vemos cada vez más lejano el sentido común y las decisiones se toman a través de normas legales que buscan regular las situaciones de la vida cotidiana de las personas. Las certificaciones y los estándares responden, en demasiadas ocasiones, a necesidades del mercado y la economía. Se trata de un entorno en el que la norma y la economía se imponen a la persona $y$, en consecuencia, se exige a la arquitectura cumplir con unos requisitos que no nacen desde las necesidades de las personas. En el ámbito universitario también sucede así, por lo que en este módulo se invitó a reflexionar sobre cómo impulsar y fomentar la soberanía de las personas en la educación universitaria.

Se recibieron un total de 50 resúmenes que fueron valorados por el Comité Científico. ${ }^{6}$ Posteriormente, de los resúmenes aceptados, se recibieron 28 artículos que fueron presentados a modo de comunicación en el Congreso.
Figura 1: Fotografía de logotipo del Congreso Arcadia5 diseñado por IDk (http://www.liquiddinamik.liquidmaps. org). Fuente: Elaboración propia.
6 Irati Labaien Egiguren (Instituto Hegoa), Josep Maria Llop (Càtedra UNESCO- Lleida), Sandra Bestraten Castells (Universidad Politécnica de Cataluña), Col.lectiu punt 6 (Barcelona), Elia Gutiérrez Mozo (Universidad de Alicante), Vicente Javier Díaz García (ETSAUniversidad de Las Palmas de Gran Canaria), Paz Núñez Martí (Universidad de Alcalá de Henares), Roberto Goycoolea Prado (ETSA-Universidad de Alcalá de Henares), Ligia Nunes (ASF Portugal), Isabel Raposo (Universidade Técnica de Lisboa), Eva Álvarez Isidro (ETSA-Universidad Politécnica de Valencia), Plácido Lizancos Mora (ETSAC Universidade A Coruña), Joseba Sainz de Murieta Mangado (Ingeniería Sin Fronteras), Esteban de Manuel Jerez (ETSAS-Universidad de Sevilla), Alex Mitxelena Etxeberria (ETSA UPV/EHU), Izaskun Aseguinolaza Braga (ETSA UPV/EHU), Koldo Telleria Andueza (ETSA UPV/EHU), Margaret Bullen (HEFA. UPV/EHU), Belén Gesto Barroso $(\mathrm{ICHaB})$. 


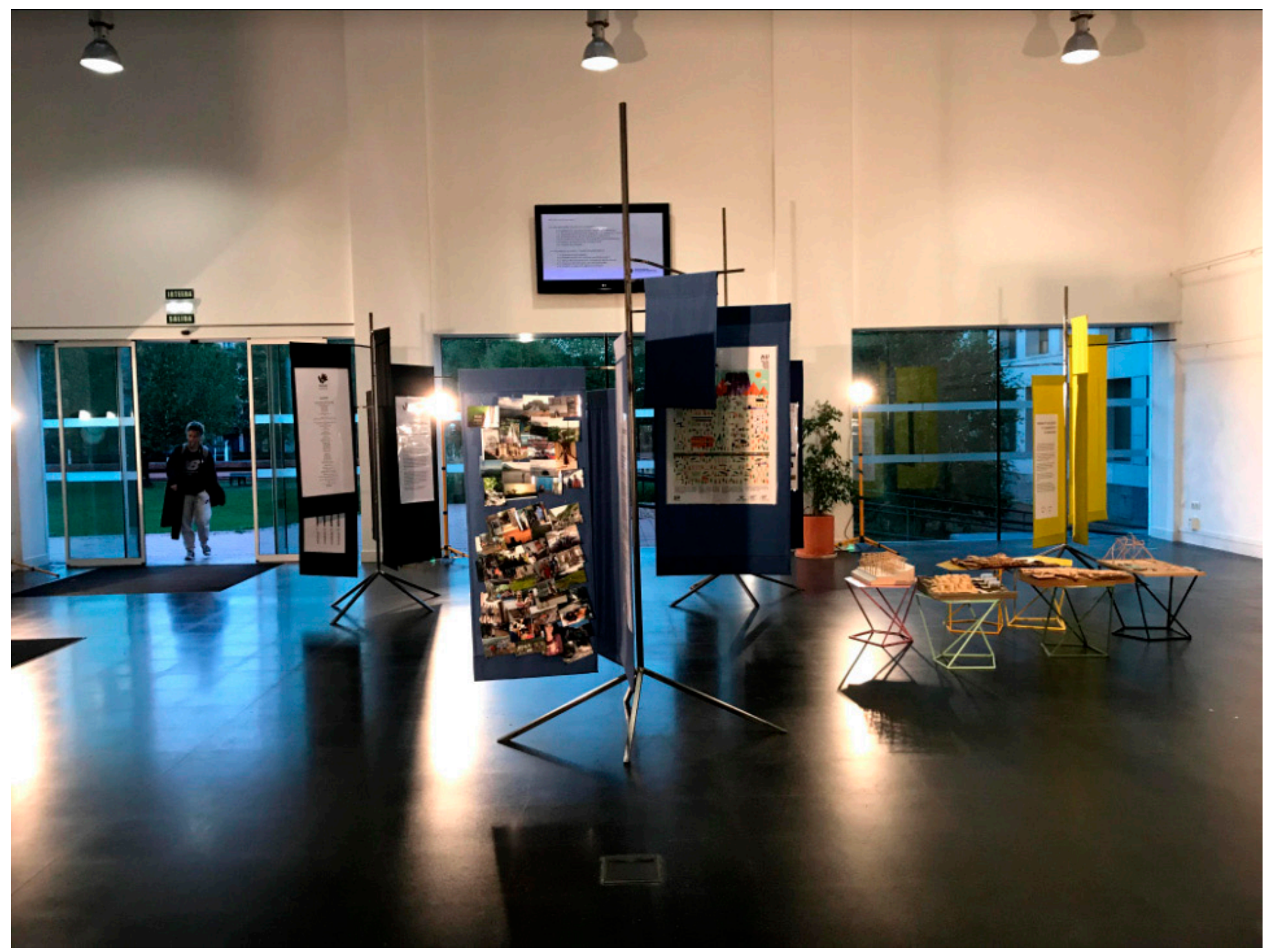

Figura 2: Fotografía de una zona de las exposiciones de Arcadia5. Fuente: Elaboración propia.
7 El proyecto Gaztenpatia es una iniciativa de Euskal Fondoa y la Universidad del País Vasco/Euskal Herriko Unibertsitatea (UPV/EHU) que posibilita al alumnado universitario realizar sus Prácticas, curriculares o voluntarias, y sus Trabajos Fin de Grado (TFG) en los proyectos que desarrollan las organizaciones y entidades socias de Euskal Fondoa en El Salvador, Nicaragua y Guatemala.

8 Arquitecta (1973) por la E.T.S. de Friburgo, Suiza, y Doctora (2007) por la TU-Delf en los Países Bajos. Fue Secretaria General de Habitat International Coalition (HIC) de 2003 a 2013. Es defensora de los derechos humanos, luchando por la dignidad humana en el territorio y la ciudad. Es autora de varios libros y artículos.
Se pudo contar con la participación de 65 personas. Entre ellas, participaron activamente Placido Lizancos, Esteban de Manuel, Julián Salas y Vicente Díaz García pero también asistió y colaboró en los diferentes eventos un nutrido grupo de asistentes formado por doctorandxs y estudiantes de grado interesadxs por la temática del Congreso.

Además, con motivo de la celebración de Arcadia5 en la E.T.S. de Arquitectura, presentamos una retrospectiva de los trabajos relacionados con la cooperación al desarrollo que se han realizado con la participación del alumnado y profesorado de la Escuela. La exposición, que se encuentra disponible, quedó formada por: Trabajos Fin de Grado realizados en los últimos años relacionados con la cooperación al desarrollo; paneles que recogen las experiencias del alumnado de la Escuela participante en el proyecto Gaztenpatia ${ }^{7}$ de la UPV/EHU; paneles que resumen el Proyecto de Innovación Educativa llevado a cabo durante los cursos 2014-15 y 2015-16 con el fin de incorporar competencias transversales que permitan una formación integral de las personas que cursan el Grado de Fundamentos de Arquitectura; el proyecto maCCOa llevado a cabo en Mozambique; y trabajos llevados a cabo en contextos de cooperación al desarrollo en la asignatura de Proyectos Arquitectónicos IX (Figura 2).

Cada uno de los módulos fue presentado por una ponencia invitada. Para ello, pudimos contar con Ana Sugranyes ${ }^{8}$ que tituló su intervención "Hacia el hábitat del buen vivir" (Figura 3), Teresa del Valle 


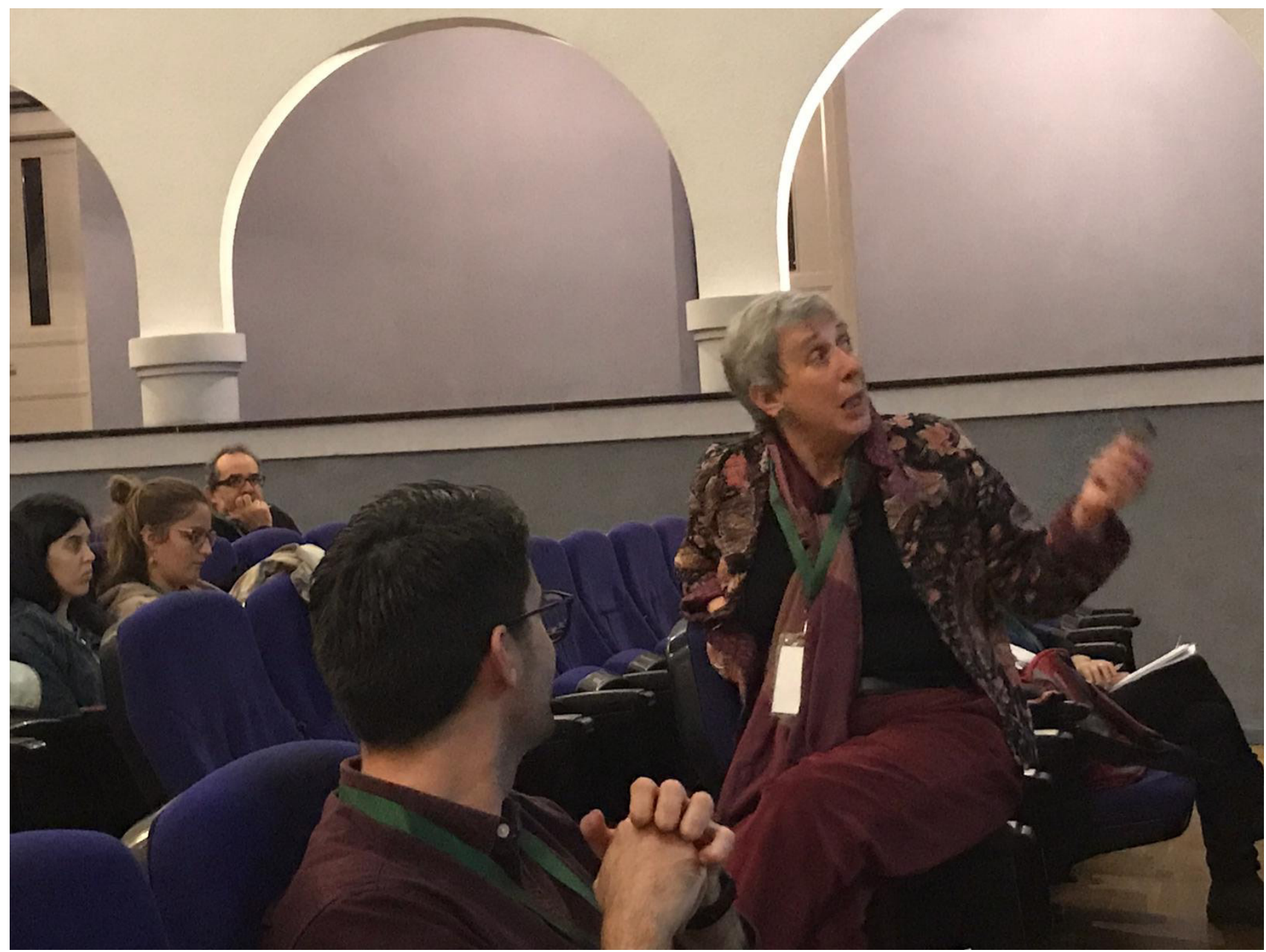

Murga $^{9}$ con una ponencia titulada "El espacio desde la movilidad libre y segura, y la evocación", Iñigo Hernández Aycart, ${ }^{10}$ antiguo alumno de la Escuela, que presentó su experiencia en Argentina con el título "Arquitectura en la escasez" y Luis Cabedo Mas ${ }^{11}$ que, a través de su exposición "El aprendizaje-servicio como oportunidad para trabajar competencias de compromiso social en la docencia universitaria", nos hizo reflexionar sobre el tema del "aprendizaje-servicio".

Las aportaciones, tanto de lxs ponentes como de una selección de los artículos presentados, se recogerán en el libro que se encuentra en preparación y que se prevé publicar a finales de 2019.

Al final de cada uno de los módulos se habilitó un espacio para una dinámica de tipo "world cafe" (Figura 4). Un tiempo para el diálogo y la reflexión en el que pudieron expresarse todas las personas participantes en el Congreso. Un momento para el intercambio de opiniones e ideas sobre lo escuchado en cada módulo.

En el primero de estos encuentros informales se compartió la necesidad de poner en marcha políticas urbanas más integrales, más allá de atender a los problemas urbanos a través de la dotación de viviendas. Por otra parte, también se reivindicó la necesidad de que la Universidad adopte una postura más activa hacia la sociedad: en concreto, que las escuelas de arquitectura proporcionen una educación más interdisciplinar que permita abordar la complejidad del medio urbano. Además, se insistió en la necesidad de que la cooperación al desarrollo en la universidad se desvincule del voluntariado y se inserte en el desarrollo curricular del grado.
Figura 3: Ana Sugranyes durante su ponencia en Arcadia5. Fuente: Elaboración propia

9 Doctora en Antropología por la UPV/EHU. Fue la primera profesora y después la primera Catedrática de Antropología de la UPV/EHU. Pionera en la Universidad en el campo de la Antropología feminista. Es miembro de Jakiunde, Academia de las Ciencias, de las Artes y de las Letras vasca y pertenece al grupo fundador de la European Association of Social Anthropologists. Es premio Emakunde en 2010 y premio Eusko Ikaskuntza en mayo de 2018. Ha escrito varios libros y numerosos artículos.

10 Arquitecto por la E.T.S. de Arquitectura de la UPV/EHU en 2015. Teniendo en cuenta su juventud, su experiencia en el ámbito de la cooperación al desarrollo es dilatada. En TECHO Argentina es coordinador del área de Investigación y Desarrollo, cuyo objetivo es desarrollar soluciones de vivienda de emergencia, adaptadas a las necesidades tanto de las familias destinatarias como del voluntariado y del propio TECHO. 


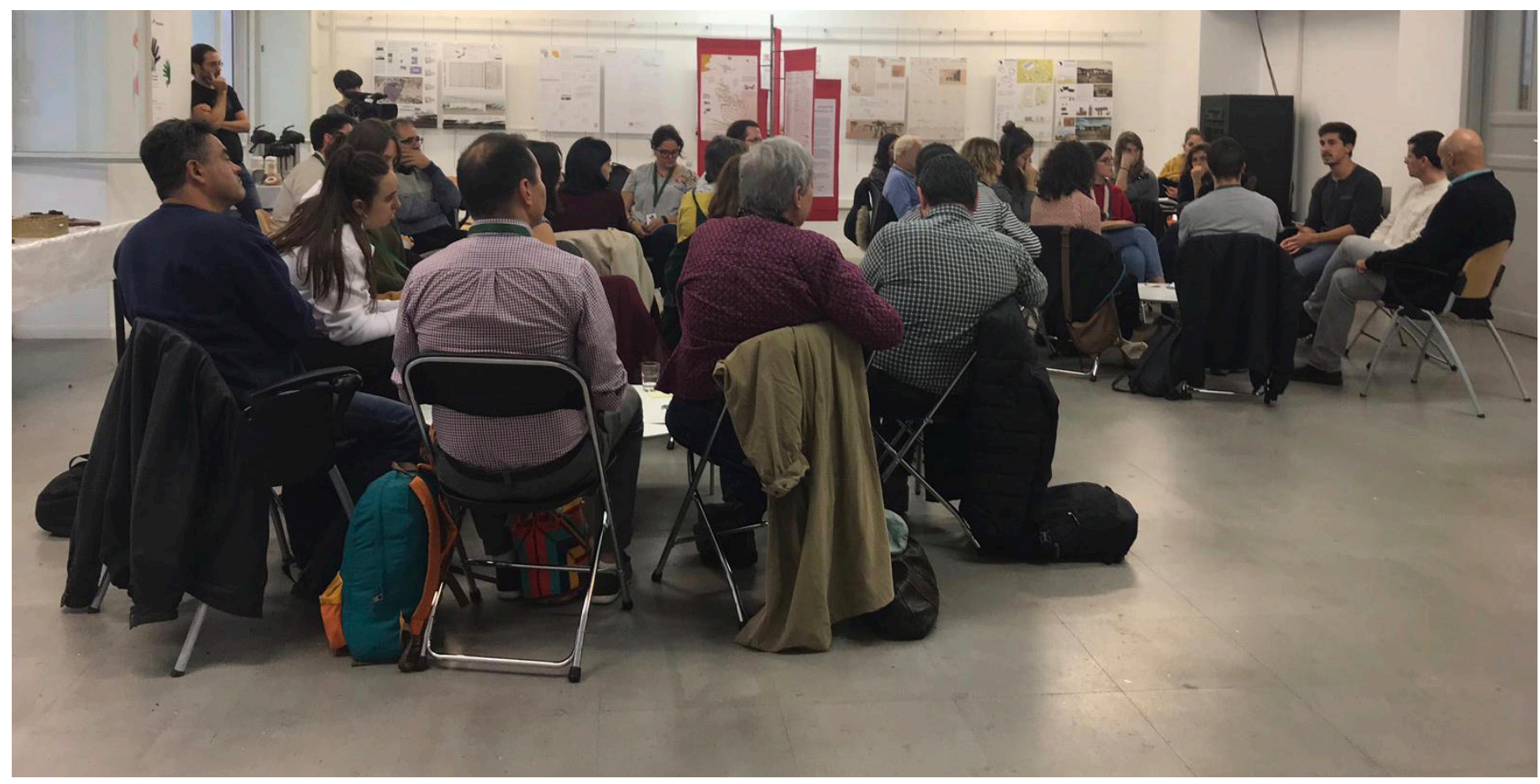

Figura 4: Una de las conversaciones world café que tuvieron lugar en Arcadia5. Fuente: Elaboración propia.

Figura 5: Otra las conversaciones world café. Fuente: Elaboración propia.

11 Doctor Ingeniero químico y profesor contratado doctor de la Universidad Jaume I con una dilatada trayectoria en el mundo de la Química. Es autor de más de 45 publicaciones científicas en revistas internacionales y más de 60 aportaciones a congresos, varios capítulos de libro, dos patentes y co-fundador de una spin-off (Nanobiomatters S.L.). Además, coordina el Grupo de Innovación Educativa en Ciencia de los Materiales (GIE-CEM) y su labor en la innovación educativa se centra en el aprendizaje basado en proyectos y el aprendizaje-servicio (Service Learning).
En el segundo, el módulo "Entorno Humano", se trató el derecho al habitar y el derecho a la movilidad, el compromiso a largo plazo de todas las personas implicadas en el desarrollo de proyectos de cooperación, la reciprocidad existente entre las personas que se relacionan en proyectos de este tipo y, por ello, se recalcó la importancia de la escucha y la empatía necesarias para abordar estos proyectos.

En el tercero, se habló de la población y de las formas de vida, se centró gran parte del debate alrededor de la vivienda. Se expusieron ejemplos de viviendas progresivas, que pueden ser construidas según surjan las necesidades o las posibilidades de las personas que las habitan y se remarcó la dificultad de conseguir que las viviendas construidas sean duraderas. También se habló de habitar la vivienda por parte de una unidad de convivencia o por parte de una colectividad, y de la necesidad de disponer también espacios colectivos. Así, se citaron el

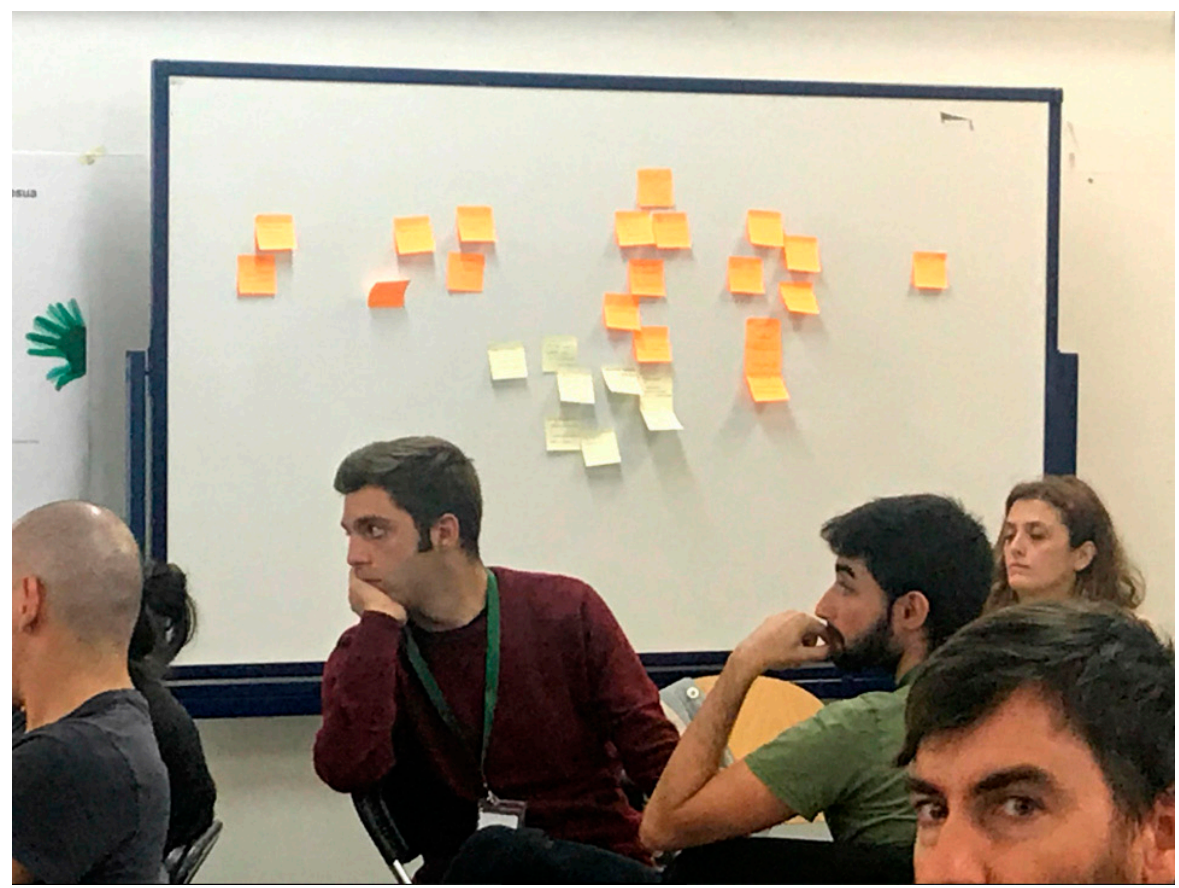




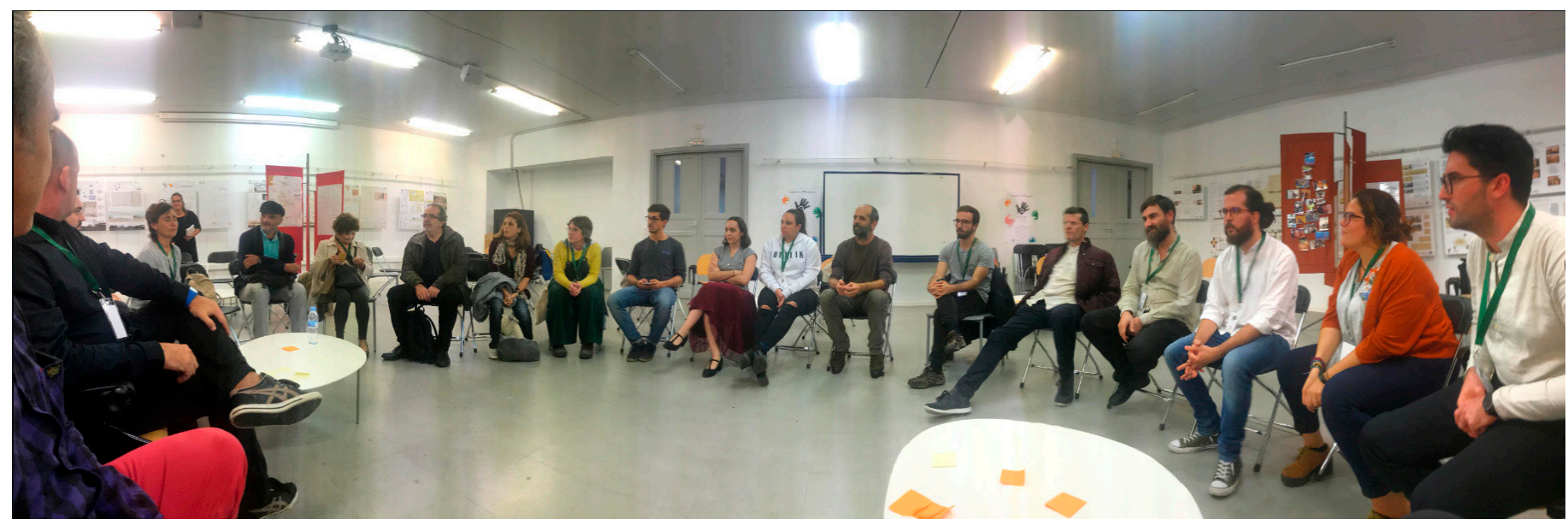

modelo cooperativo uruguayo y el cooperativismo catalán. Por último, se señaló la necesidad de explorar nuevas tipologías, así como la posibilidad de adaptar las existentes (Figura 5).

Tras las comunicaciones del último módulo y finalizadas las aportaciones de lxs asistentes, el diálogo del último "world café" se centró en recoger impresiones y sensaciones generales del conjunto del Congreso. A modo de conclusión se recogieron palabras, conceptos e ideas como "más poder", "inspiración", "los problemas de allí son de aquí", "+ empatía", "mucho que hacer", "más praxis en la uni", "colaboración", "ilusión de ser muchas", "pilas cargadas", "reflexiones" y "subidón" (Figura 6).

Con Arcadia5 se cumplen 10 años del inicio de esta andadura. En su trayectoria se ha ido construyendo un corpus de conocimiento importante que debe ser parte de las escuelas de arquitectura, formando nuevxs arquitectxs capaces de mirar al mundo, desde lo local y lo pequeño, pero sin olvidarse de lo global y de cómo estamos interconectadxs unxs con otrxs; centrándose en las personas y sus necesidades, cuidando el medio ambiente.

Para ello, seguiremos trabajando y nos encontraremos en Arcadia6. Nos vemos en Barcelona.
Figura 6: El world café final que cerró Arcadia5. Fuente: Elaboración propia. 


\section{Bibliografía}

De Manuel Jerez, Esteban y Martín Hernández, Manuel J. (2010). Jornadas de Arquitectura y Cooperación al Desarrollo. Hábitat y Sociedad, 1, 175-179. DOI: 10.12795/HabitatySociedad.2010.i1.13.

Gómez Genua, Enkarni, Mitxelena Etxeberria,Alex y Aseguinolaza Braga, Izaskun (2017). Proyecto de Innovación Educativa (PIE) "La Cooperación al Desarrollo como marco para la adquisición de competencias trasversales en el Grado en Fundamentos de Arquitectura”. En Caridad Yáñez, Eduardo et al.(eds.), ARCADIA4. Congreso de Arquitectura y Cooperación al Desarrollo (pp. 67-81). La Coruña: Universidad Da Coruña.
CEURI (Comité Español Universitario de Relaciones Internacionales) (2006). Código de Conducta de las Universidades en materia de Cooperación al Desarrollo. España: CRUE (Conferencia de Rectores de las Universidades Españolas). Recuperado el 8 de septiembre de 2019 de: http:/ /www.ocud.es/es/files/ doc512/codigoconducta.pdf.

Pennese, Claudia y Mitxelena, Alex (eds.) 2015. El proyecto maCOOa. Arquitectura y urbanismo en cooperación al desarrollo. Leioa: Servicio Editorial UPV/EHU.

Mitxelena Etxeberria, Alex et al. (2019). Arcadia5. Arquitectura y hábitat. Soberanía de las personas. Hábitat y Sociedad, 12, 135142.

<http://dx.doi.org/10.12795/HabitatySociedad.2019.i12.09> 